\title{
Renal transplantations from parents to siblings with autosomal recessive Alport syndrome caused by a rearrangement in an intronic antisense $A l u$ element in the COLAA3 gene led to different outcomes
}

\author{
Jun-ya Kaimori • Naotsugu Ichimaru • Yoshitaka Isaka Fusako Hashimoto $\cdot$ Xuejun Fu $\cdot$ Yuya Hashimura \\ Hiroshi Kaito · Kazumoto Iijima • Masahiro Kyo • Tomoko Namba • Yoshitsugu Obi • Masaki Hatanaka • \\ Isao Matsui · Yoshitsugu Takabatake $\cdot$ Masayoshi Okumi $\cdot$ Koji Yazawa $\cdot$ Norio Nonomura • \\ Hiromi Rakugi • Shiro Takahara
}

Received: 2 July 2012/ Accepted: 4 November 2012/Published online: 9 December 2012

(C) Japanese Society of Nephrology 2012

\begin{abstract}
Two siblings with autosomal recessive Alport syndrome (ARAS) obtained renal transplants from their consanguineous parents. Their COLAA3 mRNA transcripts were disrupted by a 139 bp intronic sequence between exon 48 and 49, which was derived from an antisense $A l u$ element in this intron. The new amino acid sequence from the cryptic exon was terminated by a stop codon at the 1511 th codon, resulting in the loss of $76 \% \alpha 3$ (IV)NC1. This is the first case report of kidney transplantations between ARAS-homozygous siblings and their heterozygous parents. The brother experienced acute rejection just after transplantation and post-transplantation anti-
\end{abstract}

J. Kaimori · N. Ichimaru $(\square) \cdot$ S. Takahara

Department of Advanced Technology for Transplantation,

Osaka University Graduate School of Medicine,

2-2 J8 Yamadaoka, Suita, Osaka 565-0871, Japan

e-mail: ichimaru@att.med.osaka-u.ac.jp

J. Kaimori · Y. Isaka ( $₫) \cdot$ T. Namba · Y. Obi ·

M. Hatanaka - I. Matsui - Y. Takabatake - H. Rakugi

Department of Geriatrics and Nephrology, Osaka University

Graduate School of Medicine, 2-2 B6 Yamadaoka, Suita,

Osaka 565-0871, Japan

e-mail: isaka@kid.med.osaka-u.ac.jp

F. Hashimoto $\cdot$ X. Fu $\cdot$ Y. Hashimura $\cdot$ H. Kaito $\cdot$ K. Iijima Department of Pediatrics, Kobe University Graduate School of Medicine, Kobe, Japan

M. Kyo

Sakurabashi Iseikai Clinic, Osaka, Japan

M. Okumi · K. Yazawa · N. Nonomura

Department of Specific Organ Regulation (Urology),

Osaka University Graduate School of Medicine, Suita,

Osaka, Japan glomerular basement membrane (GBM) nephritis, whereas the sister has experienced no problems to date. The antiGBM nephritis could have resulted from the acute rejection. The COLAA3 gene heterozygous mutated parents, who are possibly at risk for thin basement membrane disease, have maintained their renal functions without urinary abnormalities after renal transplantation to date.

Keywords Alport syndrome - Anti-GBM nephritis · Cryptic exon $\cdot$ Renal transplantation

\section{Introduction}

Alport syndrome (AS) is a genetic glomerular nephritis that progresses to end stage renal disease (ESRD) and is associated with sensorineural hearing loss and ocular lesions [1]. Most families with AS show an X-linked dominant inheritance pattern, with mutations in the COLAA5 gene. By contrast, autosomal recessive AS (ARAS) may be responsible for $10 \%$ of the total number of cases, and is induced by mutations in the COLAA3 and COLAA4 genes [2]. A small fraction of Alport patients undergoing renal transplantation develop anti-glomerular basement membrane (GBM) nephritis, which ultimately results in ESRD [3]. Here, we describe the first case report of renal transplantations from heterozygous carrier parents to ARAShomozygous siblings. Their mutation resides in an intronic Alu element, and causes a cryptic exon in COL4A3 transcripts, resulting in the loss of almost all of the of $\alpha 3(I V) N C 1$ domain. The brother, whose HLA type has two mismatches with that of his father, experienced acute rejection after the operation, resulting in the loss of his allograft through post-transplantation anti-GBM nephritis. 


\section{Case report}

Genetic analysis

Blood samples from the siblings (brother and sister) and their consanguineous parents were subjected to genotyping analyses with the approval of the ethics committee of Kobe University Graduate School of Medicine (\#301) and the informed consent of this family. No mutation was found in the COLAA4 and COLAA5 genes of these family members. However, the $C O L A A 3$ transcripts of this family were disrupted by a 139 bp insertion at the junction of exons 48 and 49, which derived from a 293 bp antisense Alu element located in intron 48-49. This insertion was spliced into the $\alpha 3$ (IV) mRNA because of a $\mathrm{C}-\mathrm{G}$ transversion activating a cryptic acceptor splice site in the element (Fig. 1). The new amino acid sequence from the cryptic exon was SHFHHPGWSAVVQSWLTAASTS, which started at the 1489th codon in the mutated COL3A4 (p.T1489sfsX23), and the premature termination resulted in a protein of 1510 amino acids (compared to 1670 amino acids in the wild type) and the loss of $76 \%$ of the $\alpha 3$ (IV)NC1 domain (183 C-terminal amino acids) in the mutated COL4A3 protein. Genotyping of the family revealed that the siblings were homozygotes and the parents heterozygotes. From these analyses, we concluded that the siblings had ARAS and that their parents were heterozygous carriers. This presented a very rare opportunity for renal transplantations from heterozygous carrier parents to ARAS siblings.
The brother's case

Hematuria was observed at the age of two. At five he developed sensorineural deafness, and at ten he was diagnosed with AS by kidney biopsy. He started hemodialysis at the age of 19 , with a decline in his renal function. Three months later, a living donor renal transplantation operation was performed with his father - who had no hematuria or proteinuria - as a donor. Their blood types matched, but there was an HLA type mismatch at one B and one DR locus. The lymphocyte cross match test was negative. His postoperative time course is shown in Fig. 2. Cyclosporin A (CsA), mizoribine (Miz), prednisolone, and antithymocyte globulin (ATG) were given as immunosuppressants. He experienced a sudden rise in serum creatinine at the seventh day postoperation. A kidney biopsy showed acute rejection. Orthoclone OKT3 and pulses of prednisolone were given with the suspensions of CsA and Miz. His creatinine returned to $1.5 \mathrm{mg} / \mathrm{dl}$ at discharge. One year post-transplantation, slight proteinuria and hematuria were noted, which worsened after 16 months. A renal biopsy performed after 18 months showed IgG and C3 at GBM and also glomerular crescent formation (Fig. 3) [Banff classification: borderline change (g0, t1, i1, v0, ah0, ah0), CSAassociated tubulopathy, mild]. His serum anti-GBM antibody level was $1327 \mathrm{EU} / \mathrm{ml}$. He was diagnosed with anti-GBM nephritis. His creatinine level increased to around $5 \mathrm{mg} / \mathrm{dl}$ and he returned to hemodialysis therapy at 20 months posttransplantation, despite receiving pulses of prednisolone and deoxyspergualin therapy. He is currently waiting for a
Fig. 1 a Top: schematic presentation of COLAA3 intron 48-49 containing an antisense Alu element. The blank box indicates the limits of the abnormally spliced Alu fragment in these patients. Dotted lines indicate the mRNA resulting from the abnormal splicing. Bottom: DNA and amino acid sequence of the cryptic exon between exon 48 and 49 , including the new 22 amino acid sequence and a termination codon. b Left: pedigree of this family. The parents are heterozygous, whereas the two affected siblings are homozygous for the mutated allele. Right: sequence of the intron 48-49 Alu element. The acceptor and donor splice sites with the $\mathrm{C}-\mathrm{G}$ substitution are indicated by red rectangles
A

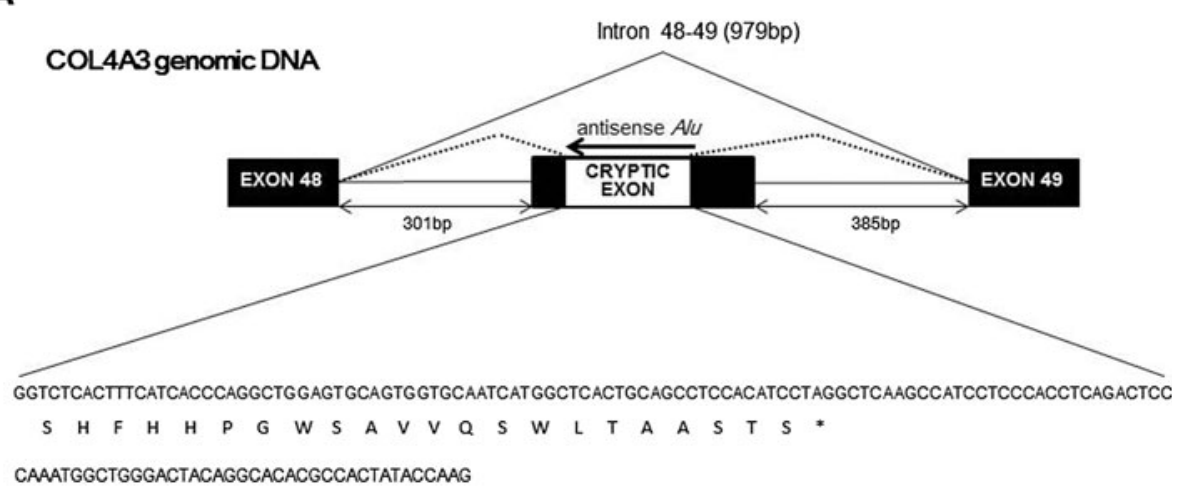

B

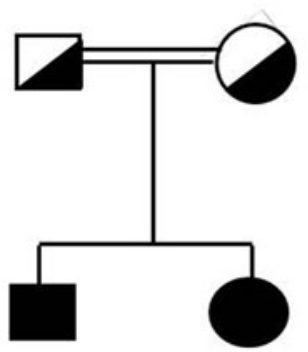

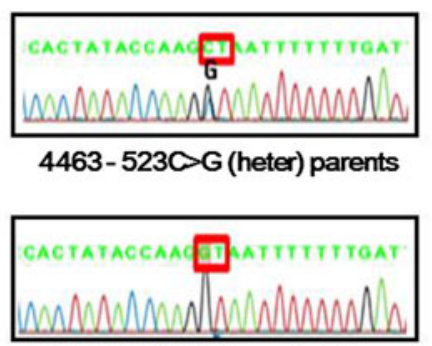

4463-523C $>$ G (homo) siblings 
Fig. 2 Schematic presentation of time course of the results for the brother after renal transplantation from his father. $R B$ renal biopsy, $M P$ methylprednisolone, $C s A$ cyclosporin A, Miz mizoribine, Pred prednisolone, Aza azathioprine, $U$-pro urine protein, $O B$ occult blood, black dots show the patient's serum creatinine level
Fig. 3 Pictures of the allograft renal biopsy specimen in the case of the brother's transplantation. a PAS staining of a $1 \mathrm{hr}$ biopsy of the allograft, with virtually no abnormalities $(\times 200)$. b PAS staining of a biopsy of the allograft 18 months after the transplantation. The image shows glomerular crescent formation and massive infiltration of mononuclear cells (×200). c PAM staining of a biopsy of the allograft 18 months after the transplantation. This image also shows glomerular crescent formation $(\times 200)$
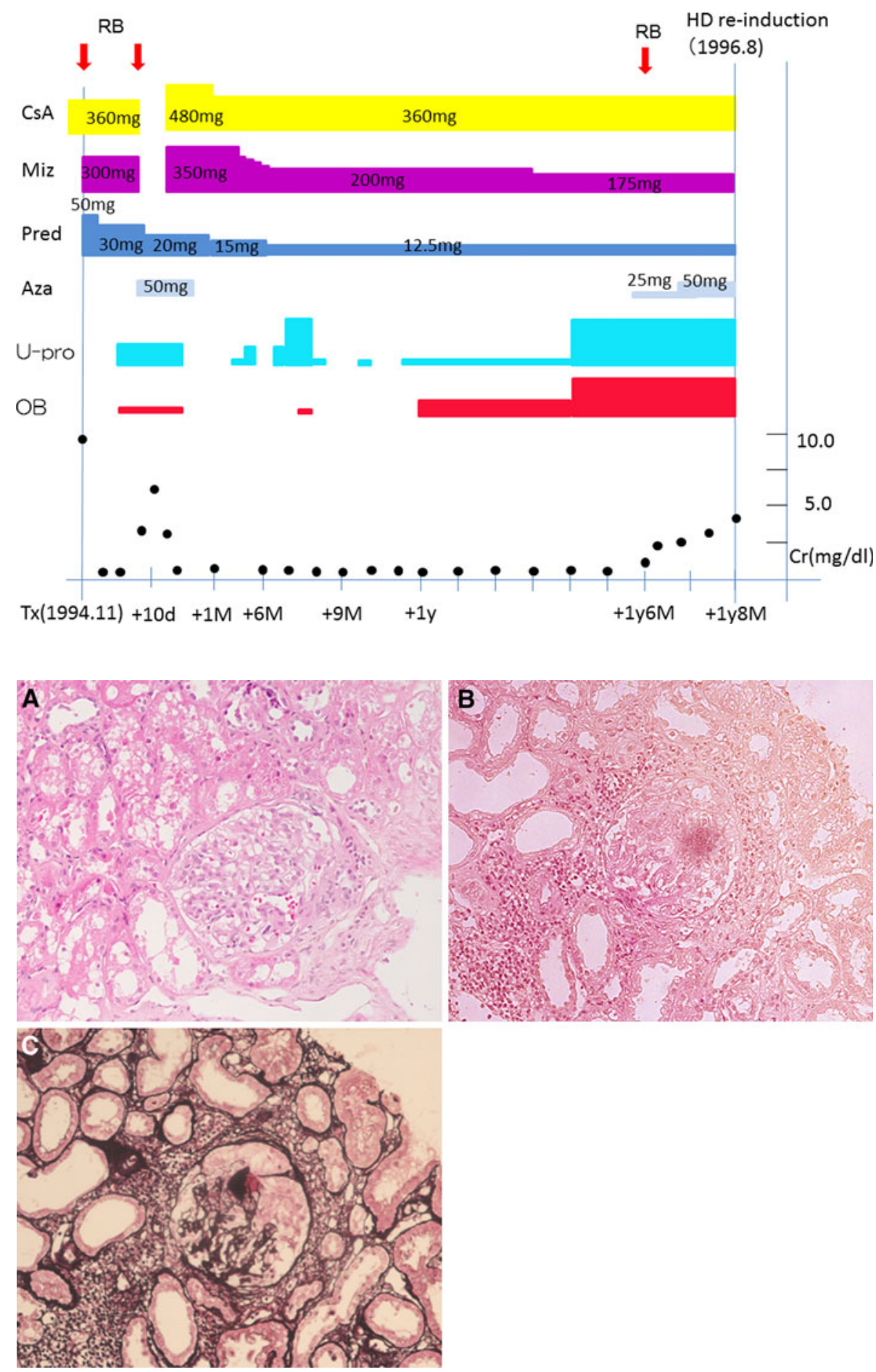

deceased donor renal transplantation whilst receiving hemodialysis. His serum anti-GBM antibody level is now less than $10 \mathrm{EU} / \mathrm{ml}$.

The sister's case

In her childhood, hematuria, proteinuria, and sensorineural deafness were noted. She started continuous ambulatory peritoneal dialysis therapy at the age of 21 . She also had ocular lesions. An ABO-incompatible renal transplantation was performed with her mother as the living donor. The mother had no urinary abnormalities before the transplantation. Plasma exchange and splenectomy were performed before the operation to remove antibodies. HLA loci were completely matched. The lymphocyte cross match test was negative. Her immunosuppressants were tacrolimus, 
prednisolone, and mycophenolate mofetil. To date, she has experienced no acute rejection or anti-GBM nephritis, and has a creatinine level of $0.8 \mathrm{mg} / \mathrm{dl}$. Her serum anti-GBM antibody level is less than $10 \mathrm{EU} / \mathrm{ml}$.

The outcomes of the parent donors

We followed up their renal symptoms for long periods (the 65-year-old father for 18 years, and the 66-year-old mother for 10 years). During these periods, their renal functions were maintained without any urinary abnormalities.

\section{Discussion}

This is only the second report of ARAS induced by the genome rearrangement of an intronic antisense Alu element harboring a cryptic exon in the COL4A3 transcript [4]. However, the mutation site and insertion size of our case are completely different and novel. One of the mechanisms that causes major gene rearrangements in humans involves Alu elements, which are interspersed repetitive sequences comprising approximately $10 \%$ of the human genome. They are considered to be retrotransposable and to have significant roles in genomic evolution and human genetic diseases. Type IV collagen molecules are heterotrimers built by the folding of three $\alpha$ chains, as induced by interactions between their NC1 domains [5]. Thus, it is predicted that the absence of almost all of the $\mathrm{NC} 1$ domain would prevent the mutant $\alpha 3$ (IV) chain from forming heterotrimers and from incorporating into the GBM.

It is interesting that only the brother developed antiGBM nephritis. It is possible that the brother's acute rejection induced the anti-GBM nephritis by exposing the relevant antigens, as previously hypothesized [6]. The consanguineous marriage of the parents induced two haplotype matches to the mother in the sister's HLA genes that would have prevented acute rejection from occurring in her allograft. In addition, potent immunosuppressive agents such as tacrolimus and mycophenolate mofetil had beneficial effects on the sister's graft outcome.

There is currently no evidence that asymptomatic carriers of COLAA3 and COLAA4 mutations are at a greater risk of developing ESRD than the general population; caution should be taken when using asymptomatic carriers of COLAA3 and COLAA4 mutations as renal transplantation donors. This is because heterozygous mutation in COLAA3 and COLAA4 induces thin basement membrane nephropathy (TBMN), and some of the TBMN patients progress to ESRD later in their lives. According to Gross et al. [7], $33 \%$ of living donors with heterozygous mutations in COLAA genes experienced substantial renal function loss after transplantation. Therefore, careful evaluation of donors is needed to minimize the risks to donor and recipient. In the current Japanese transplantation environment, dialysis patients have a very low probability of receiving deceased donor allografts; therefore, we were obliged to conduct renal transplantations from asymptomatic carriers of mutations as living donors. Fortunately, the parents have experienced no urine abnormalities, and have maintained their renal function.

\section{Conclusion}

We experienced the renal transplantations of two siblings with ARAS. The brother experienced post-transplantation anti-GBM nephritis following the loss of his allograft, whereas the sister has experienced no problems to date.

Acknowledgments This study was supported in part by a Grant-inAid for Progressive Renal Disease Research from the Ministry of Health, Labor, and Welfare of Japan.

Conflict of interest The results presented in this paper have not been published previously in whole or part, except in abstract format. The authors have no commercial associations that might pose a conflict of interest in connection with the submitted manuscript.

\section{Reference}

1. Gregory MC, Shamshirsaz AA, Kamgar M, Bekheirnia MR. Alport syndrome, Fabry disease, and nail-patella syndrome. In: Schrier RW, editor. Diseases of the kidney and urinary tract, vol I. 8th ed. Philadelphia: Lippincott Williams \& Wilkins; 2007. p. 540-69.

2. Lemmink HH, Mochizuki T, van den Heuvel LP, Schroder $\mathrm{CH}$, Barrientos A, Monnens LA, van Oost BA, Brunner HG, Reeders ST, Smeets HJ. Mutations in the type IV collagen alpha 3 (COL4A3) gene in autosomal recessive Alport syndrome. Hum Mol Genet. 1994;3:1269-73.

3. Kashtan CE. Renal transplantation in patients with Alport syndrome. Pediatr Transpl. 2006;10:651-7.

4. Knebelmann B, Forestier L, Drouot L, Quinones S, Chuet C, Benessy F, Saus J, Antignac C. Splice-mediated insertion of an Alu sequence in the COL4A3 mRNA causing autosomal recessive Alport syndrome. Hum Mol Genet. 1995;4:675-9.

5. Weber S, Engel J, Wiedemann H, Glanville RW, Timpl R. Subunit structure and assembly of the globular domain of basementmembrane collagen type IV. Eur J Biochem. 1984;139:401-10.

6. vd Heuvel LP, Schroder CH, Savage CO, Menzel D, Assmann KJ, Monnens LA, Veerkamp JH. The development of anti-glomerular basement membrane nephritis in two children with Alport's syndrome after renal transplantation: characterization of the antibody target. Pediatr Nephrol. 1989;3:406-13.

7. Gross O, Weber M, Fries JW, Muller GA. Living donor kidney transplantation from relatives with mild urinary abnormalities in Alport syndrome: long-term risk, benefit and outcome. Nephrol Dial Transpl. 2009;24:1626-30. 